\title{
Gears and Belt Drives for Non-Uniform Transmission
}

\author{
Hellmuth Stachel
}

\begin{abstract}
Ordinarily, gears and belt drives are used for uniform transmission of rotations between parallel axes. Here we focus on the nonuniform case, i.e., with non-constant transmission ratio. We treat the geometry of tooth profiles and pulleys and their algorithmic computation. Concerning gears, we recall a method due to $\mathrm{S}$. Finsterwalder. Concerning belt drives, we study their relation to tooth profiles and focus on 'strict' cases which work without tightener.
\end{abstract}

Keywords Nonuniform Transmission, Planar Gearing, Belt Drives, Geometric Kinematics

\section{Introduction}

The problem of designing noncircular gears has often been addressed in publications (see [1, 2, 3, 4] and in particular Litvin's monograph [5], pp. 346-381). Such gears are used for nonuniform transmission between parallel axes. Similarly, certain cam-like mechanisms have rolling centrode contact surfaces. Here we recall an applicable, classical design method formulated by S. Finsterwalder (1862-1951, see [6], [7, p. 284] or [8, p. 205]) which is also useful for an algorithmic computation.

J. Hoschek [9] and F. Freudenstein [10] (see also [11]) created methods to design belt drives for given nonuniform transmission such that the length of the surrounding belt remains constant. Such belt drives with belt slack zero will briefly be called strict. After discussing geometric properties of belt drives we present a modification of Hoschek's method for computing conjugate pulleys for strict belt drives that is not confined to discrete (i.e., polygonal) models, like those treated in [12] or [13], but nevertheless produces satisfactory results. However implementation of large transmission ratio variations results in pulley profiles with singularities analogous to

H. Stachel

Institute of Discrete Mathematics and Geometry, Vienna University of Technology, Wiedner Hauptstr. 8-10/104, A 1040 Wien, Austria

e-mail: stachel@dmg.tuwien.ac.at 
undercut gear teeth. Hoschek's method as well as ours fail in the case of global 1:1 transmissions where a full rotation of the input wheel corresponds to a full rotation of the output wheel.

\section{Gearing for Nonuniform Transmission}

Let the driving wheel $\Sigma_{1}$ rotate about the center $O_{1}=01$ through the angle $\varphi_{1}$ with respect to the frame of the fixed housing $\Sigma_{0}$ while the output wheel $\Sigma_{2}$ rotates about $O_{2}=02$ through $\varphi_{2}$. Then the pole 12 of the relative motion $\Sigma_{2} / \Sigma_{1}$ divides the segment $O_{1} O_{2}$ in the ratio of instantaneous angular velocities, i.e., $O_{1} 12: O_{2} 12=\dot{\varphi}_{2}: \dot{\varphi}_{1}=\omega_{2}: \omega_{1}$.

In the sequel we seek gears and belt drives that transmit rotary motion according to some transmission function

$$
\varphi_{2}=f\left(\varphi_{1}\right) \text { for } \quad 0 \leq \varphi_{1} \leq 2 \pi
$$

Function $f\left(\varphi_{1}\right)$ is assumed to be strictly monotonic, quite often differentiable, and obeys $f\left(\varphi_{1}+2 \pi\right)=f\left(\varphi_{1}\right)+2 \pi / n$ for $, n \in \mathbb{Z}, n \neq 0$. The integer $n$ is called global transmission ratio. The transmission function $f\left(\varphi_{1}\right)$ defines the associated perturbation function $g\left(\varphi_{1}\right)$ by

$$
g\left(\varphi_{1}\right)=n \cdot f\left(\varphi_{1}\right)-\varphi_{1} \quad \text { or } \quad f\left(\varphi_{1}\right)=\left[\varphi_{1}+g\left(\varphi_{1}\right)\right] / n
$$

Because of $g\left(\varphi_{1}+2 \pi\right)=g\left(\varphi_{1}\right)$ function $g\left(\varphi_{1}\right)$ is periodic and can be set up as a Fourier series. In the gear box $\Sigma_{0}$ we use a coordinate frame (see Fig. 3) with $O_{1}=(0,0), O_{2}=(e, 0)$, and $12=\left(r_{1}\left(\varphi_{1}\right), 0\right)$. Then the coordinate $r_{1}\left(\varphi_{1}\right)$ of 12 obeys $\omega_{2}: \omega_{1}=r_{1}:\left(r_{1}-e\right)$, hence

$$
r_{1}\left(\varphi_{1}\right)=\frac{e f^{\prime}\left(\varphi_{1}\right)}{f^{\prime}\left(\varphi_{1}\right)-1}=\frac{e\left(1+g^{\prime}\left(\varphi_{1}\right)\right)}{1-n+g^{\prime}\left(\varphi_{1}\right)}
$$

when the prime indicates differentiation by $\varphi_{1}$.

$c_{1} \subset \Sigma_{1}$ and $c_{2} \subset \Sigma_{2}$ are conjugate tooth profiles if and only if they are an enveloping pair of the relative motion $\Sigma_{2} / \Sigma_{1}$. Due to the 'Law of Gearing' the common normal line at the meshing point $C$ passes always through the relative pole 12 (Fig. 1). We express the position of $C$ in polar coordinates $(\rho, \psi)$ with respect to the relative pole 12 and choose the negative vector of the pole velocity as zero-axis for measuring the polar angle $\psi$. We may suppose $0 \leq \psi \leq \pi$ for $\rho \in \mathbb{R}$. Angle $\psi$ with the meshing normal is unique even for $\rho=0$. As the two polodes $p_{1}, p_{2}$ are in contact at 12 , these coordinates of $C$ are the same with respect to $\Sigma_{1}$ and $\Sigma_{2}$. 


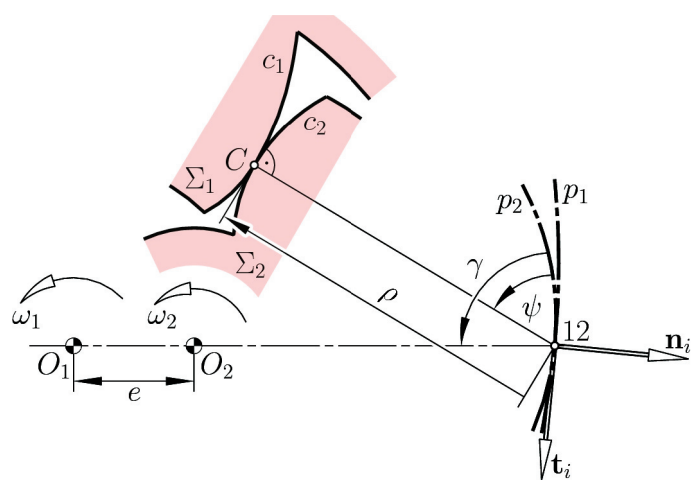

Fig. 1 Conjugate tooth profiles $c_{1}, c_{2}$ for the relative polodes $p_{1}, p_{2}$

Let $\left(\mathbf{t}_{i}, \mathbf{n}_{i}\right)$ denote the Frenet frame of the polode $p_{i}, i=1$, 2, with position vector $\mathbf{p}_{i}(t)$ and arc length $s$. The derivatives by time $t$ are

$$
\dot{\mathbf{t}}_{i}=v \kappa_{i} \mathbf{n}_{i}, \dot{\mathbf{n}}_{i}=-v \kappa_{i} \dot{\mathbf{t}}_{i} \quad \text { and } \quad \dot{\mathbf{p}}_{i}=v \mathbf{t}_{i}
$$

with pole velocity $v=\mathrm{d} s / \mathrm{d} t$ and curvature $\kappa_{i}$ of $p_{i}$. The position vector $\mathbf{c}_{i}=$ $\mathbf{p}_{i}+\rho\left(\cos \psi \mathbf{t}_{i}+\sin \psi \mathbf{n}_{i}\right)$ of the tooth profile has the velocity

$\dot{\mathbf{c}}_{i}=v \mathbf{t}_{i}+\dot{\rho}\left(\cos \psi \mathbf{t}_{i}+\sin \psi \mathbf{n}_{i}\right)+\rho \dot{\psi}\left(-\sin \psi \mathbf{t}_{i}+\cos \psi \mathbf{n}_{i}\right)+\rho v \kappa_{i}\left(\cos \psi \mathbf{n}_{i}-\sin \psi \mathbf{t}_{i}\right)$

orthogonal to $\mathbf{c}_{i}-\mathbf{p}_{i}$. This implies

$$
\dot{\rho}=-v \cos \psi
$$

This differential equation for $\mathbf{c}_{i}(t)$ is independent of the curvature $\kappa_{i}$ of the polodes $p_{i}$ and is therefore the basis for

S. Finsterwalder's principle of gearing [6, p. 243]: ${ }^{1}$ We imagine $p_{1}$ as a flexible metal band and replace $c_{1}$ by a discrete set of line elements, each attached to the polode $p_{1}$ by fixing the angle $\psi$ and the distance $\rho$ (Fig. 2). Then for any flex $p_{2}$ of $p_{1}$ the curve $c_{2}$ formed by the attached line elements is conjugate to $c_{1}$ if the relative motion $\Sigma_{2} / \Sigma_{1}$ is defined by $p_{2}$ rolling along $p_{1}$. This principle works not only for wheels rotating about fixed centers, but for any planar motion given by a pair of polodes.

In the sense of Differential Geometry the functions and obeying (2) can be called natural functions of the specified gearing.

\footnotetext{
1 This is the discretized version of the general Reuleaux (or Camus) principle saying that conjugate profiles are envelopes of any curve $c_{0}$ while an auxiliary curve atached to $c_{0}$ is rolling on the polodes.
} 


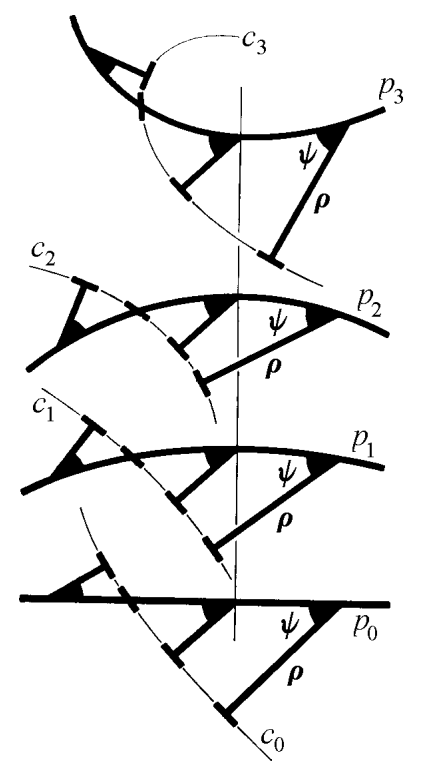

Fig. 2 S. Finsterwalder's principle of gearing (extracted from [7], p. 284, Fig. 446, compare [8], Abb. 151)

If the metal band is stretched like $p_{0}$ in Fig. 2 then is the tooth profile of the conjugate broaching rack. A straight line segment defines a constant angle $\psi$ and therefore the nonuniform involute gearing.

Theorem 1. The following conditions are necessary to avoid local undercuts of conjugate tooth profiles given by their natural functions:

$\left(1+v \kappa_{i}\right) \rho-v \sin \psi \neq 0$ for

$i=1,2$ and $\dot{\psi} \neq \rho-v \sin \psi$.

The proof is based on Frenet equations of the tooth profile $c_{i}$. The first condition excludes singularities, the second avoids intersections between $c_{1}$ and $c_{2}$ because of 3-point contact.

For computing conjugate tooth profiles $c_{1}, c_{2}$ of a given transmission one has to take the following steps:

(1) Compute the polode $p_{1}$ in $\Sigma_{1}$ with polar coordinates $\left(r_{1},-\varphi_{1}\right)$ and $r_{1}$ by (1) as well as $p_{2}$ in $\Sigma_{2}$ with coordinates $p_{2}=\left(r_{1}-e,-\varphi_{2}\right)$.

(2) Rectify $p_{1}$, i.e., bend it into the straight line $p_{0}$. Freely choose the rack tooth profile $c_{0}$ as long as it is nowhere orthogonal to $p_{0}$ and compute the polar coordinates $(\rho(s), \psi(s))$ with respect to $p_{0}$.

(3) Bend $p_{0}$ back into $p_{1}$ and $p_{2}$ and use $(\rho, \psi)$ with respect to the Frenet frames of $p_{1}$ and $p_{2}$ for obtaining $c_{1}$ and $c_{2}$. 
(4) The applicable segments of $c_{1}$ and $c_{2}$ are arrived at by inspecting their relative movement in view of local and global undercuts.

\section{Geometry of Belt Drives}

Let $b_{1}, b_{2}$ be conjugate pulley profiles (Fig. 3). The upper belt span between the contact points $B_{1} \in b_{1}$ and $B_{2} \in b_{2}$ defines a new system $\Sigma_{3}$. As line $b_{3}=B_{1} B_{2}$ rolls on $b_{1}$ and $b_{2}$, the points $B_{1}, B_{2}$ are the relative poles 13 and 23, resp., and this implies the necessary condition $[10,9,14,8]$.

Theorem 2. label2 At each instant the upper belt span must be aligned with the relative pole 12 .

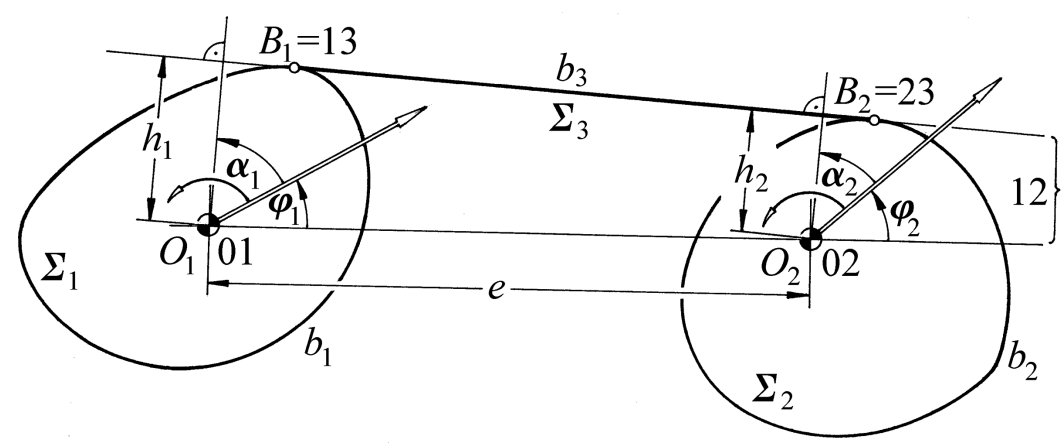

Fig. 3 The upper belt span connects the relative poles 13 and 23

Under the relative motion $\Sigma_{3} / \Sigma_{1}$ an arbitrary point $C$ attached to the line $B_{1} B_{2}$ traces an involute $c_{1}$ of $b_{1}$ (see Fig. 4). The path of $C \in \Sigma_{3}$ under $\Sigma_{3} / \Sigma_{2}$ is an involute $c_{2}$ in $\Sigma_{2}$, which contacts $c_{1}$ under $\Sigma_{2} / \Sigma_{1}$ at $C$.

Theorem 3. Conjugate pulley profiles $b_{1} \subset \Sigma_{1}$ and $b_{2} \subset \Sigma_{2}$ produce the given transmission from $\Sigma_{1}$ to $\Sigma_{2}$ if and only if $b_{2}$ and $b_{1}$ are evolutes of an enveloping pair $\left(c_{2}, c_{1}\right)$ of the relative motion $\Sigma_{2} / \Sigma_{1}$. At each instant the endpoints $B_{2}$ and $B_{1}$ of the upper belt span are corresponding under the curvature transformation of the relative motion $\Sigma_{2} / \Sigma_{1}$ (see Fig. 4).

Of course, $b_{1}$ and $b_{2}$ must be closed curves - contrary to the locally acting tooth profiles $c_{1}$ and $c_{2}$.

Now we recall Finsterwalder's method with the polodes $p_{1}, p_{2}$ as metal bands. This time we focus on the envelopes of the attached normal lines making the oriented angle $\psi$ with the polodes. These envelopes $b_{1}, b_{2}$ are conjugate pulley profiles. Hence we obtain

Theorem 4. For any transmission function and any driving pulley $b_{1}$ there is a unique conjugate profile $b_{2}$. However, $b_{2}$ needs not be convex. 


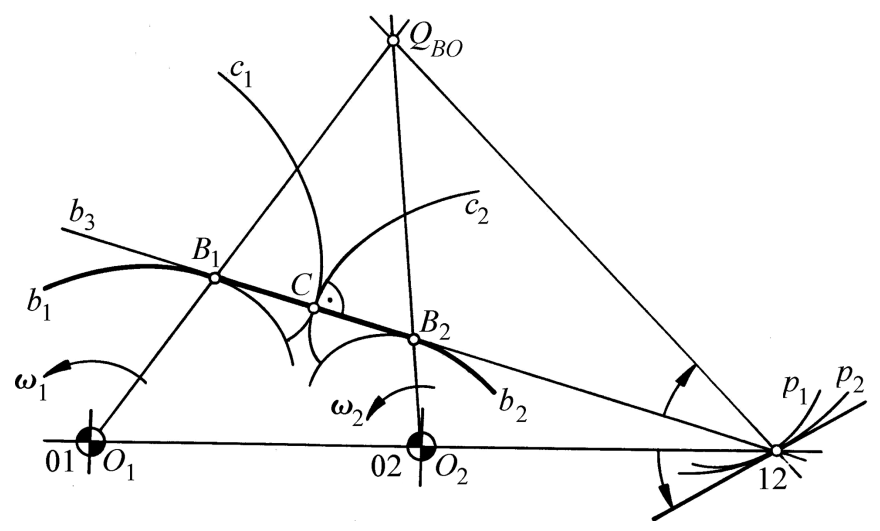

Fig. 4 Points $B_{2} \mapsto B_{1}$ and $O_{2} \mapsto O_{1}$ are corresponding under the curvature transformation of $\Sigma_{2} / \Sigma_{1}$ (Bobillier's construction, see [14], Abb. 152)

We call $\psi(s)$ the natural angle function of this design. The important case $\psi=$ const. of involute gearing corresponds to evolutoides ${ }^{2}$ of the polodes as conjugate pulley profiles $b_{1}, b_{2}$.

\section{Strict Belt Drives}

When in each position the length of the surrounding belt with taut spans remains constant, then the lower belt span rolls on $b_{1}$ and $b_{2}$, too.

Theorem 5. [9]: Conjugate pulley profiles $b_{1}$ and $b_{2}$ operate without needing a tightener if and only if at each instant both the upper and the lower belt span are aligned with the relative pole 12 (Fig. 5)

Wunderlich and Zenow [15] discovered 1975 the following nontrivial example of a nonuniformly transmitting strict belt drive for $n=-1: \Sigma_{2} / \Sigma_{1}$ is a line-symmetric motion with ellipses as polodes $p_{1}$ and $p_{2}$. The pulleys are ellipses $b_{1}, b_{2}$ confocal with $p_{1}, p_{2}$ (Theorem of Graves). It should be noted that in the uniform case with $n=1$ any convex disk together with a translated copy yield a strict belt drive.

Theorem 5 implies an algorithm for computing strict belt drives:

(1) In an arbitrary initial position $\left(\varphi_{1}=\varphi_{1}^{(0)}\right)$ we specify an upper belt span $(\psi=$ $\left.\psi^{(0)}\right)$ passing through 12 . We attach this line to $\Sigma_{1}$.

(2) The next point of intersection of this line with the polode $p_{1}$ defines a position $\left(\varphi_{1}=\varphi_{1}^{(1)}\right)$ where this line becomes a lower belt span. This must be tangent to the conjugate profile $b_{2}$, and we attach it to $\Sigma_{2}$.

\footnotetext{
${ }^{2}$ An evolutoide of curve $p$ is the envelope of lines meeting $p$ at a constant angle $\psi$.
} 


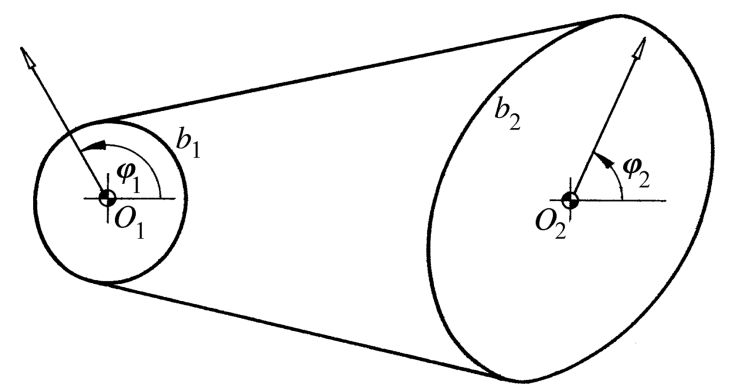

Fig. 5 A strict belt drive for the transmission function $\varphi_{2}=0.5\left[\varphi_{1}+0.2 \sin \varphi_{1}+0.16 \sin 2 \varphi_{1}+\right.$ $\left.0.008 \cos 2 \varphi_{1}\right]$. The length of the surrounding belt varies within about $0.002 \%$

(3) When its next point of intersection with $p_{2}$ becomes the relative pole $\left(\varphi_{1}=\varphi_{1}^{(2)}\right)$, the line again covers an upper belt span. In general, this gives a new tangent line of $b_{1}\left(\psi=\psi^{(2)}\right)$.

Iteration gives a finite set of lines tangent to $b_{1}$. In contrast to Hoschek's method using Bézier curves we represent the tangent lines by their support function $h_{1}\left(\alpha_{1}\right)$ (see Fig. 3). Then we use a least square method for finding the Fourier series of fixed order which approximates the computed tangent lines best. By Theorem 4 we obtain the conjugate $c_{2}$.

This algorithm works well (see Fig. 5) in all examples with global transmission ratio $n \neq 1$. The computed lines obviously envelope a unique curve ${ }_{1}$. This observation together with some arguments give rise to

Conjecture 1: For a given nonuniform transmission function with global transmission ratio $n \neq 1$ there is a one-parametric set of conjugate pairs $\left(b_{1}, b_{2}\right)$ of profiles for a strict belt drive. However, these profiles are convex $\left(h_{i}+h_{i}^{\prime \prime}>0\right.$ by [16]) only if the given transmission lies sufficiently close to the uniform transmission with the same global ratio $n$.

A rigorous mathematical proof is open but there is some supporting evidence: For convex relative polodes and an analytic transmission function the mapping $\left(\varphi_{1}^{(0)}, \psi^{(0)}\right) \mapsto\left(\varphi_{1}^{(2)}, \psi^{(2)}\right)$ of lines is analytic, too. Lines tangent to $p_{1}(\psi=0)$ are fixed. Passing through $O_{1}(\psi=\gamma$, see Fig. 1) is preserved. And the support function obeys

$$
\begin{aligned}
& h_{1}\left(\varphi_{1}^{(2)}\right): h_{1}\left(\varphi_{1}^{(0)}\right)=\Omega\left(\varphi_{1}^{(2)}\right): \Omega\left(\varphi_{1}^{(1)}\right) \quad \text { with } \\
& \Omega=\omega_{2} / \omega_{1}
\end{aligned}
$$

The excluded case $n=1$ shows a strange behavior that was observed -but not reported - by J. Hoschek: The lines obtained by the above algorithm do not envelope any curve. It is proved in [17] that in this case a starting line passing through $O_{1}$ 
after iteration does not rotate fully about $O_{1}$ but approaches a limiting position. By continuity, this seems to contradict the required convexity of $b_{1}$ and leads to

Conjecture 2: There is no pure belt drive for nonuniform transmission with global transmission ratio $n=1$.

\section{Conclusion}

Tooth profiles for gears and pulleys of belt drives are closely related. However, the determination of strict belt drives for a given non-uniform transmission leads to deeper mathematical problems. It is to hope that in the near future the conjectures stated above can be proved rigorously.

\section{References}

1. Biing-Wen B., Computer aided design of elliptical gears with circular-arc teeth, Mechanism and Machine Theory, Vol. 39, 2004, pp. 153-168.

2. Chang S.-L., Tsay C.-B., Wu L.-I., Mathematical model and undercutting-analysis of elliptical gears generated by rack cutters. Mechanism and Machine Theory, Vol. 31, 1996, pp. 879-890.

3. Peters R.M., Analysis and synthesis of eccentric chainwheel drives, Mechanism and Machine Theory, Vol. 7, 1972, pp. 111-119.

4. Spitas V., Costopoulos T., Spitas C., Fast modeling of conjugate gear tooth profiles using discrete presentation by involute segments, Mechanism and Machine Theory, Vol. 42, 2007, pp. 751-762.

5. Litvin F.L., Gear Geometry and Applied Theory. Prentice Hall, Englewood Cliffs, NJ, 1994.

6. Baier O., Über die Abstandsempfindlichkeit ebener Verzahnungen. Konstruktion, Jg. 5, Heft 8, 1953, pp. 242-245.

7. Hohenberg F., Konstruktive Geometrie in der Technik. 2. Aufl., Springer-Verlag, Wien, 1961.

8. Wunderlich W., Ebene Kinematik. Bibliographisches Institut, Mannheim 1970.

9. Hoschek J., Konstruktion von Kettengetrieben mit veränderlicher Übersetzung mit Hilfe von Bézier-Kurven. Forsch. Ing,-Wes., Vol. 48, 1982, pp. 81-87.

10. Freudenstein F., Ch.-K. Chen., Variable-ratio chain drives with noncircular sprockets and minimum slack - theory and application, Journal of Mechanical Design, Vol. 113, 1991, pp. $253-262$.

11. Fraulob S., Nagel T., Ungleichförmig übersetzende und hochübersetzende ZahnriemenGetriebe. VDI-Berichte 1845, 2004, pp. 249-261.

12. Xin C.L., Jian L., Research on the mapping models and designing methods of variable-ratio chain/belt drives, Mechanism and Machine Theory, Vol. 37, 2002, pp. 955-970.

13. Wang H., Wang Sh., Wang Y., Design and machinery of non-circular sprocket's tooth, Proceedings of the 9th World Congress on the Theory of Machines and Mechanisms, Milano 1995, Vol. 1, pp. 556-559.

14. Tidwell P.H., Bandukwala N., Dhande C.F., Reinholtz C.F., Webb G., Synthesis of Wrapping Cams, Transactions of the ASME, Vol. 116, 1994, pp. 634-638.

15. Wunderlich W., Zenov P., Contribution to the geometry of elliptic gears, Mechanism and Machine Theory, Vol. 10, 1975, pp. 273-278.

16. Strubecker K., Differentialgeometrie I. Sammlung Göschen, Walter de Gruyter, Berlin, 1964.

17. Stachel H., Nonuniform chain-wheel drives, Proceedings of the 8th World Congress on the Theory of Machines and Mechanisms, Prague 1991, Vol. 5, pp. 1343-1346. 